\title{
REPORT ON \\ TOPICS IN THE THEORY OF DIVERGENT SERIES*
}

BY W. A. HURWITZ

1. Introduction. It is with some reluctance that I have acceded to the request of the programme committee to address the Society on the present status of problems concerning divergent series, since three admirable expository treatments of this field have already been given to the Society and published in the Bulletin, by W. B. Ford, $\dagger$ R. D. Carmichael, $\ddagger$ and C. N. Moore. $\S$ In particular Professor Carmichael has so closely followed the trend which my own thoughts have taken (except that I should not have presented them so elegantly) as to make it difficult for me to give an adequate account without a good deal of repetition. At any rate I shall take advantage of his paper in order to plunge in medias res today, and also to omit references to original sources unless these seem especially desirable.

If we have the symbol

$$
\Sigma u_{n}=u_{1}+u_{2}+u_{3}+\cdots
$$

we define

so that

$$
x_{n}=u_{1}+u_{2}+\cdots+u_{n},
$$

In case

$$
u_{1}=x_{1} ; \quad u_{n}=x_{n}-x_{n-1}, \quad n>1 \text {. }
$$

$$
\lim _{n \rightarrow \infty} x_{n}
$$

exists, we say that the series $\Sigma u_{n}$ or the sequence $\left(x_{n}\right)$ is convergent, the limit of the sequence being the value or sum of the series. The importane of this concception lies in the fact that many formal transformations carried out on infinite series as if they are finite sums can be proved correct. Instances arose early in the study of series, however, in which

*Presented before the Society at the Symposium held in New York City, April 23, 1921.

†'This Bulletin, vol. 25 (1918-19), p. 1.

$\ddagger$ Ibid., vol. 25 (1918-19), p. 97 .

\$ Ibid., vol. 25 (1918-19), p. 258. 
such transformations, though assuredly not correct on the basis of existing theory, nevertheless led in special cases to correct results.* The attempt to justify such results led naturally to generalizations of the notion of the value of a series.

I shall denote methods of definition of value by capital letters $A, B, \cdots$; I shall express the fact that a series or sequence can be evaluated by a method $A$ by saying that it is summable $A$; and the resulting value I shall denote by $A \mathrm{~L}\left(x_{n}\right)$.

2. Linearity and Regularity. One of the important properties of definitions is that of linearity. A definition $A$ is said to be linear if

$$
A \mathbf{L}\left(x_{n}+x_{n}{ }^{\prime}\right)=A \mathbf{L}\left(x_{n}\right)+A \mathbf{L}\left(x_{n}{ }^{\prime}\right)
$$

whenever $A \mathbf{L}\left(x_{n}\right)$ and $A \mathrm{~L}\left(x_{n}{ }^{\prime}\right)$ exist, and

$$
A \mathbf{L}\left(c x_{n}\right)=c A \mathbf{L}\left(x_{n}\right)
$$

whenever $A \mathbf{L}\left(x_{n}\right)$ exists.

I have stated this property first of all, because it is the only one which is satisfied, so far as I know, by every definition which has ever been proposed as practically useful. Of even wider importance of course, although not satisfied by every useful definition, is the following. A definition $A$ is said to be regular in case it evaluates every convergent sequence, giving it the value to which it converges; that is, in case

implies $A \mathbf{L}\left(x_{n}\right)=l$.

$$
\lim _{n \rightarrow \infty} x_{n}=l
$$

Nearly every definition that has been proposed can be thrown into the following form:

Let a point set $T$ be given in space of any number of dimensions, real or complex, having a limit point $t_{0}$ (actual or symbolic $\dagger$ ) not belonging to $T$, and let the functions $a_{k}(t)[k=1$, $2, \cdots]$ be defined in $T$; then if the sequence $\left(x_{n}\right)$ is such that

$$
y(t)=\sum_{k=1}^{\infty} a_{k}(t) x_{k}
$$

\footnotetext{
* It is only necessary to allude to the interesting history of the series $1-1+1-1+\cdots$.

$\dagger$ I.e., having one or more of its coordinates infinite.
} 
converges for each $t$ in $T$ and if

$$
\lim _{t \rightarrow t_{0}(T)} y(t)=l,
$$

the sequence $\left(x_{n}\right)$ is said to be summable $G$, and $G \mathbf{L}\left(x_{n}\right)=l$. This definition could of course be expressed in terms of $u_{n}$ instead of $x_{n}$.

An important special case is that in which $T$ consists of the positive real integers and in which $a_{k}(t)$, which may then be written $a_{n k}$, is zero for $k>n$. In this case the requirement of convergence of $G$ is met automatically, and we may say:

The sequence $\left(x_{n}\right)$ is summable $G^{\prime}$, and $G^{\prime} \mathbf{L}\left(x_{n}\right)=l$ provided

where

$$
\lim _{n \rightarrow \infty} y_{n}=l \text {, }
$$

$$
y_{n}=\sum_{k=1}^{n} a_{n k} x_{k} .
$$

An especial convenience in dealing with this case is that the transformation $G^{\prime}$ may be treated by the usual algebraic machinery of linear transformations, since the first $n y$ 's depend only on the first $n x$ 's. We may speak of sums, numerical multiples, products, and powers of $G^{\prime}$. The definition of ordinary convergence is one such case; it is given by the transformation

$$
y_{n}=x_{n} .
$$

Any transformation of the form $G^{\prime}$ will have an inverse provided $a_{n n} \neq 0$ for every $n$.

For both $G$ and $G^{\prime}$ it is obvious that the condition of linearity is satisfied. Sufficient conditions for the regularity of $G$ were given by Silverman;* they were proved to be also necessary (even in a slightly more general case) by Toeplitz: $\dagger$

For the definition $G^{\prime}$ to be regular it is necessary and sufficient that

$$
\begin{aligned}
& \text { for each } k, \lim _{n \rightarrow \infty} a_{n k}=0 ; \\
& \text { for all } n, \sum_{k=1}^{n}\left|a_{n k}\right| \text { is bounded; }
\end{aligned}
$$

* Ph.D. thesis, Missouri, 1910; University of Missouri Studies, Mathematics Series, vol. 1 , no. 1 (1913).

$\dagger$ PRACE MATEMATYCZNO-FIZYCZNE, vol. 22 (1911), p. 113. 


$$
\lim _{n \rightarrow \infty} \sum_{k=1}^{n} a_{n k}=1 \text {. }
$$

The corresponding theorem for the more general definition $G$ is due to Carmichael* and Hildebrandt; $\dagger$ it has recently been restated by J. Schur: $\ddagger$

For the definition $G$ to be regular it is necessary and sufficient that

(G1) for each $k, \lim _{t \rightarrow t_{\alpha}(T)} a_{k}(t)=0$;

(G2) for each $t$ in $T, \sum_{k=1}^{\infty}\left|a_{k}(t)\right|$ converges,

and for all $t$ in $T, \sum_{k=1}^{\infty}\left|a_{k}(t)\right|$ is bounded;

$$
\lim _{t \rightarrow t_{0}\left(T^{T}\right)} \sum_{k=1}^{\infty} a_{k}(t)=1 .
$$

It should be noted that if we restrict all quantities appearing in these theorems to be real, the theorems remain true, not only as regards sufficiency, but also as regards necessity.

3. Definitions of Summability. I shall now give examples of definitions, illustrating each by application to the series

$$
1+x+x^{2}+x^{3}+\cdots
$$

For this series $u_{n}=x^{n-1}$; hence

$$
x_{n}=\frac{1-x^{n}}{1-x}, \quad x \neq 1 .
$$

As the definitions are all to satisfy the requirement of linearity, we can prove that $A \mathrm{~L}\left(x_{n}\right)=1 /(1-x)$ by showing that $A \mathrm{~L}\left(x^{n}\right)=0$. We shall therefore consider instead of (5)

$$
x_{n}=x^{n} \text {. }
$$

The usual definition of limit gives the desired result when and only when $|x|<1$. Hölder employed the transformation

$$
y_{n}=\frac{x_{1}+x_{2}+\cdots+x_{n}}{n} \text {. }
$$

* Loc. cit., p. 118.

$\dagger$ Abstract, this Bulletin, vol. 24 (1917-18), p. 429. Compare also the statement made by Carmichael in the preceding reference.

‡ Journal FÜr Mathematik, vol. 151 (1920), p. 82. 
Here, in the notation of $G^{\prime}, a_{n k}=1 / n$. This definition is obviously regular. It constitutes the first Hölder mean. The further Hölder means are conveniently described as algebraic powers of $M: M^{2}, M^{3}, \cdots$.

In order to apply $M$ to (6), we have $x_{n}=x^{n}$, and for $x \neq 1$,

$$
\begin{aligned}
y_{n} & =\frac{x+x^{2}+\cdots+x^{n}}{n}=\frac{x\left(1-x^{n}\right)}{n(1-x)} \\
& =\frac{x}{n(1-x)}-\frac{x}{1-x} \frac{x^{n}}{n} .
\end{aligned}
$$

The first term approaches zero as $n$ becomes infinite, and

$$
\lim _{n \rightarrow \infty}\left|\frac{x^{n}}{n}\right|=\left\{\begin{array}{cc}
0, & |x| \leqq 1 \\
\infty, & |x|>1 ;
\end{array}\right.
$$

hence (4) is summable $M$ to the value $1 /(1-x)$ for $|x| \leqq 1$, $x \neq 1$, and is not summable $M$ for $|x|>1$. I shall defer until later the consideration of the behavior for $x=1$. $^{*}$

Hence $M$ evaluates (4) to the value which would be expected, not only at points inside the circle of convergence, but also at all points on the circle other than $x=1$; and at no points outside the circle. It can readily be shown that for any value of $r, M^{r}$ accomplishes no more for the series (4).

Cesàro gave the formulas:

$$
C_{1}=M \text {; }
$$

(C) $\quad y_{n}=\frac{n x_{1}+(n-1) x_{2}+\cdots+x_{n}}{n(n+1) / 2}$;

$\left(C_{r}\right) \quad y_{n}=\frac{n \cdots(n+r-2) x_{1}+\cdots+1 \cdots(r-1) x_{n}}{n(n+1) \cdots(n+r-1) / r}$.

For every positive integer $r, C_{r}$ is regular.

Extensions of Cesàro's method were given by Knopp and Chapman. Chapman's formula is

$$
y_{n}=\sum_{k=1}^{n} r \frac{(n-1) ! \Gamma(n+r-k)}{(n-k) ! \Gamma(n+r)} x_{k},
$$

which reduces to Cesàro's form when $r$ is a positive integer,

${ }^{*}$ See $\$ 6$. 
but has a meaning when $r$ is fractional, irrational or even complex, provided $r$ is not a negative integer or zero. In a limiting sense, it may be said to have a meaning even for $r=0: C_{0}=I$. Applying $C_{2}$, for illustration, to (6), we have $x_{n}=x^{n}$, for $x \neq 1$,

$$
\begin{aligned}
y_{n} & =\frac{n x+(n-1) x^{2}+\cdots+x^{n},}{n(n+1) / 2}, \\
(1-x)^{2} y_{n} & =\frac{n x-(n+1) x^{2}+x^{n+2}}{n(n+1) / 2} \\
& =\frac{2 x}{n+1}-\frac{2 x^{2}}{n}+\frac{2 n x^{2}}{n+1}\left(\frac{x^{n}}{n^{2}}\right) .
\end{aligned}
$$

Hence as before $C_{2} \mathrm{~L}\left(x^{n}\right)=0$ for $|x| \leqq 1, x \neq 1 ; C_{2} \mathrm{~L}\left(x^{n}\right)$ does not exist if $|x|>1$. The same result can be obtained for $C_{r}$, in the same fashion if $r$ is a positive integer, and by other considerations in any case when $\mathbf{R}(r)>0$.

It is seen that no definition of Hölder or Cesàro serves to evaluate (4) outside its region of convergence. A convenient form of definition is given by the exponential mean:*

$$
y_{n}=\sum_{k=1}^{n} \frac{(n-1) !}{(n-k) !(k-1) !} \frac{(r-1)^{n-k}}{r^{n-1}} x_{k} .
$$

The successive coefficients are the terms of the binomial expansion of $[(1-1 / r)+1 / r]^{n-1}$. The definition is regular if and only if $r$ is real and $\geqq 1$. It is readily seen that $E_{1}=I$. The name "exponential mean" is suggested because of the interesting algebraic property $E_{r} E_{s}=E_{r s}$.

Applying $E_{r}$ to (6), we have

$$
\begin{aligned}
y_{n} & =\sum_{k=1}^{n} \frac{(n-1) !}{(n-k) !(k-1) !} \frac{(r-1)^{n-k}}{r^{n-1}} x^{k} \\
& =x\left[1-\frac{1}{r}+\frac{x}{r}\right]^{n-1} .
\end{aligned}
$$

Thus $y_{n}$ will approach zero if and only if

$$
\left|1-\frac{1}{r}+\frac{x}{r}\right|<1 \text {, }
$$

* This formula was given, in slightly different notation, by Hausdorff, MATHEMATISChe ZeITsChrifT, vol. 9 (1921), p. 86; his work was unknown to me during the preparation of this paper for presentation. 
that is,

$$
|x-(1-r)|<r .
$$

The region of evaluation is therefore the interior of a circle through $x=1$, having its center at $x=1-r$. For any $r>1$, this region includes points outside the circle of convergence. For large enough $r$ any point $x$ will be obtained which satisfies the condition $\mathbf{R}(x)<1$.

A variety of definitions were given by M. Riesz. Let $\left(\lambda_{n}\right)$ be any sequence of positive numbers increasing and becoming infinite. For one form of the Riesz mean of type $(\lambda)$ and order $r$, we write (in terms of $u_{n}$ rather than $x_{n}$ )

$$
\left(R_{\lambda, r}\right) \quad y_{n}=\sum_{k=1}^{n}\left(1-\frac{\lambda_{k}}{\lambda_{n}}\right)^{r} u_{k} \text {. }
$$

For the case $\lambda_{n}=n, r=1$, this has the form

$$
\begin{aligned}
y_{n} & =u_{1}\left(1-\frac{1}{n}\right)+u_{2}\left(1-\frac{2}{n}\right)+\cdots+u_{n-1}\left(1-\frac{n-1}{n}\right) \\
& =\frac{1}{n}\left(x_{1}+\cdots+x_{n-1}\right),
\end{aligned}
$$

and is essentially the same as $M$. For any $\left(\lambda_{n}\right), R_{\lambda_{0} 0}=I$. For all cases in which $r \geqq 0, R_{\lambda, r}$ is regular.

As regards the application of $R_{\lambda, r}$ to (6), it is clear that for $\lambda_{n}=n, R_{\lambda, 1}$ gives a value at points inside and on the boundary of the circle of convergence, but nowhere outside. It can be proved that with any choice of $\left(\lambda_{n}\right)$ and $r$, evaluation of (4) outside the circle of convergence is impossible.

The definitions thus far considered have all been of type $G^{\prime}$. We consider next some which are of the more general type $G$. A definition given by Euler can readily be put in this form. Euler argued that as the series $1-1+1-1+\cdots$ is the special case, for $t=1$, of the series $1-t+t^{2}-t^{3}+\cdots$, which for $t<1$ has the value $1 /(1+t)$, therefore the former series should have the value $\frac{1}{2}$. This amounts to writing, in the case of a given series $u_{1}+u_{2}+u_{3}+\cdots$,

$$
y(t)=u_{1}+u_{2} t+u_{3} t^{2}+\cdots,
$$

and defining the value as $\lim _{t \rightarrow 1} y(t)$. This definition may be called Euler's power series method. Thrown into a form 
involving $x_{n}$ instead of $u_{n}$, it will read

$$
y_{n}=(1-t) \sum_{k=1}^{\infty} x_{k} t^{k-1}
$$

so that in the notation of $G, a_{k}(t)=(1-t) t^{k-1}$. We must of course require that the series

converge for $|t|<1$.

$$
\sum_{k=1}^{\infty} x_{k} t^{k-1}, \quad \sum_{k=1}^{\infty} u_{k} t^{k-1}
$$

We may choose to let $t$ approach 1 along real values or along some other point set $T$ in the circle $|t|<1$, having 1 as a limit point. In the first case, the fact that $P$ is regular is stated by a well known theorem due to Abel: If $\Sigma \alpha_{k} t^{k-1}$ converges, $-1<x \leqq 1$, then it is continuous even at $t=1$.

Abel's theorem was extended by Stolz and Pringsheim to the case of other point sets $T$. The conditions shown by them to be sufficient are easily shown by the theorem of Carmichael and Hildebrandt to be also necessary: $P$ is regular if $T$ lies within the angle formed by some pair of chords through $t=1$.

$P$ evaluates (4) only at points other than $x=1$, within and on the boundary of the circle of convergence. It is furthermore obvious that $P$ can never evaluate any power series at a point outside its circle of convergence, and that it is therefore of little value for the problem of analytic extension.

Several elegant methods of evaluation were given by Borel. I shall state some of them in a more general form due to Sannia.* In terms of a given sequence $\left(x_{n}\right)$, write

$$
\xi(t)=\sum_{k=1}^{\infty} x_{k} \frac{t^{k-1}}{(k-1) !}
$$

and suppose that this power series converges for all points of the plane. Call

$$
\begin{array}{rlrl}
\xi^{(0)}(t) & =\xi(t), \\
\xi^{(1)}(t) & =\frac{d}{d t} \xi^{(0)}(t), & \xi^{(-1)}(t) & =\int_{0}^{t} \xi^{(0)}(\tau) d \tau, \\
\xi^{(2)}(t) & =\frac{d}{d t} \xi^{(1)}(t), & \xi^{(-2)}(t) & =\int_{0}^{t} \xi^{(-1)}(\tau) d \tau,
\end{array}
$$

* Rendiconti di Palermo, vol. 42 (1917), p. 303. 
Define

$$
y(t)=e^{-t} \xi^{(r-1)}(t)
$$

then $\lim _{t \rightarrow \infty} y(t)$ is. to be the generalized limit of $\left(x_{n}\right)$. The cases commonly discussed are $B_{1}$, known as the Borel mean definition, and $B_{0}$, known as the Borel integral definition.

In the usual form of treatment $T$ is taken as the positive real axis, and it is shown that $B_{r}$ is always regular. It seems not to have been noted that we may allow $T$ to be a more general point set of the complex plane possessing points of arbitrarily great positive abscissas, and take the limit as $\mathbf{R}(t)$ becomes infinite. It can readily be proved that for the Borel-Sannia definition $B_{r}$ to be regular it is necessary and sufficient that all points of $T$ of sufficiently great positive abscissas lie within some parabola having its axis along the real axis and opening to the right; that is, if $t=\tau+i \sigma, \sigma^{2} / \tau$ shall be bounded.

Let us apply these results to (6). We have

$$
\begin{aligned}
& \xi^{(0)}(t)=x e^{x t}, \quad \xi^{(1)}(t)=x^{2} e^{x t}, \quad \xi^{(2)}(t)=x^{3} e^{x t}, \quad \cdots ; \\
& \xi^{(-1)}(t)=e^{x t}-1, \quad \xi^{(-2)}(t)=\frac{1}{x} e^{x t}-\frac{1}{x}-t, \quad \ldots
\end{aligned}
$$

Hence

$$
\begin{aligned}
& B_{1}: y(t)=x e^{(x-1) t} ; \quad B_{0}: y(t)=e^{(x-1) t}-e^{-t} \\
& B_{2}: y(t)=x^{2} e^{(x-1) t} ; \quad B_{-1}: y(t)=\frac{1}{x} e^{(x-1) t}-\frac{1}{x} e^{-t}-t e^{-t}
\end{aligned}
$$

Taking for $T$ the positive real axis, we see that $B_{r}$ evaluates (6) to zero for $\mathbf{R}(x)<1$, and for no other values. By appropriate choice of other sets for $T$, we can secure the evaluation for any point of the line $\mathbf{R}(x)=1$ except $x=1$.

Borel also gave a definition which he termed "absolute summability." It is not regular; it does, however, correctly evaluate all absolutely convergent series. Absolute summability has been considered important since it permitted various operations on series which were not justified by mere summability $B_{1}$ or $B_{0}$; but with Sannia's form of the Borel definitions, absolute summability becomes relatively unimportant. 
An extremely powerful definition is due to Le Roy. Write

$$
y(t)=\sum_{k=1}^{\infty} \frac{\Gamma(\overline{k-1} t+1)}{(k-1) !} u_{k},
$$

taking for $T$ the real axis near $t=1$; and $t_{0}=1$. This definition can be shown to be regular. It evaluates (4) for all points of the complex plane except $x$ real, $\geqq 1$.

The Riesz means have been extended by Hardy and Riesz so as to bring them under the form $G$. Taking the sequence $\left(\lambda_{n}\right)$ restricted as before, write

$$
\left(R_{\lambda, r}\right) \quad y(t)=t^{-r} \sum_{\lambda_{k}<t}\left(t-\lambda_{k}\right)^{r} u_{k},
$$

and let $T$ be the set of positive real numbers, $t_{0}=\infty . R_{\lambda, r}$ in this form is still regular for all $r \geqq 0$. It does not evaluate (4) in any more extended region than did the form for $R_{\lambda, r}$ previously given.

4. Relative Inclusiveness of Definitions; Equivalence. A definition, $A$, is said to include another, $B$, in case every sequence summable $B$ is summable $A$ to the same value. Two definitions are said to be equivalent if each includes the other; in this case each evaluates exactly the same sequences.

If the definitions $A, B$, whether regular or not, are of type $G^{\prime}$, $A$ includes $B$ provided there exists a regular $C$ such that $A=C B$. In case $B$ possesses an inverse, this condition may be written in the form: $A B^{-1}$ is regular; it is in this case necessary and sufficient.*

If $A, B$, whether regular or not, are of type $G^{\prime}$, they are equivalent provided there exist $C, D$, both regular, such that $A=C B$, $B=D A$. In case $A, B$ possess inverses, this condition may be written in the form: $A B^{-1}$ and $B A^{-1}$ are regular; it is in this case necessary and sufficient. $\dagger$

Certain cases fall under the criteria just given without any further investigation. For instance, since the Hölder means satisfy the condition $M^{r} M^{s}=M^{r+s}$, and since $M^{r}$ is always regular, it is clear that the Hölder mean definition of any order

* The second form of the condition is stated by Carmichael, loc. cit., p. 112 ; it has usually been applied in this form. There are cases, however, in which the first form is useful.

† Second form stated by Carmichael, loc. cit., p. 113. 
includes that of any lower order. In a similar way the exponential mean $E_{r}$ becomes more inclusive as $r$ increases, since $E_{r} E_{s}=E_{r s}$ and $E_{r}$ is regular when $r>1$.

Other instances require more investigation, but every case I have examined in which definitions of type $G^{\prime}$ are concerned depends on the criterion $I$ have stated. The Cesàro-Chapman mean $C_{r}$ includes $C_{s}$ if $\mathbf{R}(r)>\mathbf{R}(s)>-1$. In particular, for $-1<\mathbf{R}(r)<0, C_{r}$ is included in $I$; thus every series summable $C_{r},-1<\mathbf{R}(r)<0$, is convergent; and not all convergent series are summable $C_{r}$ for a fixed $r,-1<\mathbf{R}(r)<0$.

A question which has formed the starting point for a number of investigations on divergent series is the relationship of $M^{r}$ and $C_{r}$ for positive integral values of $r$. Knopp proved that for any such $r, C_{r}$ includes $M^{r}$; Schnee and Ford showed that $M^{r}$ and $C_{r}$ are actually equivalent; a number of other proofs have been given. To cite an example of the opposite kind, $M^{r}$ and $E_{s}$ are distinctly overlapping definitions; neither includes the other, except in the trivial case $M^{0}=E_{1}=I$.

It is important to point out that the method of proof of relative inclusiveness given above can be applied at times even to the comparison of definitions of different types. For in. stance, $E_{r}$ is of type $G^{\prime}$, a sequence-to-sequence transformation, while $B_{1}$ is of type $G$, a sequence-to-function transformation. But it is easily proved that $B_{1} E_{r}^{-1}=B_{1} E_{1 / r}=\mathbf{E} B_{1}$, where $\mathbf{E}$ is a function-to-function transformation:

$$
y(t)=x(r t) .
$$

Thus $B_{1}$ will include $E_{r}$ provided $\mathbf{E} B_{1}$ is regular; and this will surely be true provided $\mathbf{E}$ is regular, where the latter statement must be understood to mean that the existence of $\lim _{t \rightarrow \infty} x(t)$ is to imply the existence and equality of $\lim _{t \rightarrow \infty} y(t)$. But the regularity of $\mathbf{E}$ in the sense explained is obvious; thus $B_{1}$ includes $E_{r}$ for every $r>0$.

Into similar form may be thrown Sannia's proof that $B_{r}$ includes $B_{s}$ whenever $r<s$, and the familiar proof that $C_{r}(r>0)$ is included in $P$. On the other hand, of $M^{r}$ and $B_{1}$ neither includes the other; the same statement holds for $B_{1}$ 
and $P$. Note that no definition which evaluates a power series at a point outside its circle of convergence can include $P$.

The results for the Riesz means are interesting. As in the case of the Cesàro means, $R_{\lambda, r}$ includes $R_{\lambda, s}$ if $r>s$. We may even compare means derived from different sequences $\left(\lambda_{n}\right)$; thus, if $\mu_{n}=\log \lambda_{n}$, then $R_{\mu, r}$ includes $R_{\lambda, r}$. If $\lambda_{n}=n, R_{\lambda, r}$ is equivalent to $C_{r}$ for every $r$. In case $\lambda_{n}=e^{n}$, $R_{\lambda, r}$ is always equivalent to $I$.

5. Mutual Consistency. It has been seen that two definitions may be regular and yet such that neither includes the other. In such cases it is manifestly of the highest importance to know that the two definitions will not give different values to any sequence which each one evaluates. The circumstance in question is illustrated by an example due to Silverman.* The definitions (of type $G^{\prime}$ ):

$$
y_{n}=\frac{1}{n} \sum_{k=1}^{n} x_{k}, \quad y_{n}=\frac{1}{n} \sum_{k=2}^{n}\left[1+\frac{(-1)^{k+1}}{\log k}\right] x_{k}
$$

are both regular; but the sequence $x_{n}=(-1)^{n} \log n$ is evaluated by the first to 0 and by the second to 1 .

We call two definitions mutually consistent $\dagger$ if, whenever each of them evaluates a sequence, the two values are the same. A condition for mutual consistency can be stated as follows:

Any two definitions are mutually consistent if there exists a definition which includes each of them.

Obvious as this criterion seems, it is nevertheless of real value. An important special case is: Two definitions $A, B$ of type $G^{\prime}$ are mutually consistent if there exist $C, D$, both regular, such that $C A=D B$. In fact, the definition expressed by either of the two equal forms $C A, D B$ includes both $A$ and $B$.

Still further specialization occurs in case it happens that we can choose $C=B, D=A$. Any two regular definitions of type $G^{\prime}$ are mutually consistent if they are permutable.

* Loc. cit., p. 38.

† In a paper by Silverman and myself (Transactions of this Societr, vol. 18 (1917), p. 1), the word "consistent" was used for this idea. The term in the text, suggested by Carmichael, loc. cit., p. 111, seems preferable. 
It can be shown* that all transformations of type $G^{\prime}$ permutable with $M$ are permutable with each other; hence all definitions of type $G^{\prime}$ permutable with $M$ are mutually consistent.

An interesting case of this kind is furnished by $E_{r}$. We have seen that $E_{r}$ and $M$ are overlapping definitions; but it is easily verified that $E_{r}$ is permutable with $M$, hence $E_{r}$ and $M^{s}$ are mutually consistent.

The method outlined above, like that in the preceding section, can sometimes be applied even if the two definitions are of different types. Thus, it can be shown that $B_{1} M=\mathbf{M} B_{1}$, where $\mathbf{M}$ is the function-to-function transformation

$$
y(t)=\frac{1}{t} \int_{0}^{t} x(u) d u,
$$

and where all the formal processes involved have a meaning and are correct if applied to a sequence summable both $B_{1}$ and $M$. Thus $B_{1}$ and $M$ will be consistent if $\mathbf{M}$ is regular; that is, if the existence of $\lim _{t \rightarrow \infty} x(t)$ implies the existence and equality of $\lim _{t \rightarrow \infty} y(t)$. The transformation $\mathbf{M}$ is exactly what is known as the first Hölder (or Cesàro) mean for continuous limits; $\dagger$ it is known to be regular. Equally simple is the proof that $M^{r}$ and $B_{1}$ are mutually consistent.

This method of proof seems susceptible of wide application in studying the question of mutual consistency, which is important, and which has as yet received little attention.

6. Total Regularity. Let us now suppose we are dealing only with real sequences, and applying to them only real transformations. A regular definition must evaluate any convergent sequence, giving it its true value. It may naturally be asked whether this conservation of finite limits applies also to infinity of definite sign; in other words, whether a sequence becoming (say) positively infinite need be evaluated by a regular definition to positive infinity. The definition $2 M-I$ :

$$
y_{n}=2 \frac{x_{1}+x_{2}+\cdots+x_{n}}{n}-x_{n}
$$

is obviously regular; but if we take $x_{n}=n$, then we find

* Hurwitz and Silverman, loc. cit., p. 7.

$\dagger$ See $\$ 10$. 
$y_{n}=1$, so that

$$
\lim _{n \rightarrow \infty} x_{n}=+\infty, \quad \lim _{n \rightarrow \infty} y_{n}=1 .
$$

In this case therefore regularity does not extend to the conservation of the improper limit $+\infty$.

A regular definition may be called totally regular if it evaluates every sequence which becomes positively (negatively) infinite to $+\infty(-\infty)$.

$A$ sufficient condition that a regular definition $G$ be totally regular is that for all sufficiently great values of $k, a_{k}(t) \geqq 0$. In this case, condition (G2) of the conditions for regularity is superfluous. If the definition is of type $G^{\prime}$, the condition, $a_{n k} \geqq 0$ for all sufficiently great values of $k$, is also necessary.

A closely related consideration is that of the effect of a regular transformation on the limits of indeterminacy of a sequence which it does not evaluate. It is desirable that a definition, if it does not evaluate a specific sequence, shall at least not render its oscillation more violent; this may readily happen, however. If we apply the definition $2 M-I$ to the sequence $0,2,0,4,0,8, \cdots$, for which

we find that

$$
\liminf _{n \rightarrow \infty} x_{n}=0 \text {, }
$$

$$
\liminf _{n \rightarrow \infty} y_{n}=-\infty \text {. }
$$

Without endeavoring to answer completely the question raised, I shall merely say that the condition for total regularity is sufficient also to insure that the new limits of indeterminacy shall not fall outside the interval of the old limits.

The criterion for total regularity is easily tested for all the usual definitions. It is found that $M^{r}, C_{r}, R_{\lambda, r}$ are totally regular for the values of $r \geqq 0$ for which they are defined; $E_{r}$ is totally regular for $r \geqq 1$. When the point set $T$ is real, $P, B_{r}$ and the definition of Le Roy are totally regular.*

We may also widen the scope of the notions of relative

* The consideration of total regularity settles the question of the effect of these definitions on the series $1+x+x^{2}+\cdots$ at the point $x=1$, which was left open in $\S 3$. 
inclusiveness and equivalence to take account of sequences evaluated to $+\infty$. Obviously, on account of the relations $M^{r} M^{s}=M^{r+s}, E_{r} E_{s}=E_{r s}$, the statements previously made regarding relative inclusiveness of $M^{r}$ for varying $r$ and of $E_{r}$ for varying $r$ remain true even in the present extended sense. But it can be shown that $M^{r}$ and $C_{r}$, which are equivalent for finite limits when $r$ is a positive integer, do not retain this equivalence for the limit $+\infty$ when $r \geqq 2$; in fact there will always be sequences which are evaluated by $M^{r}$ to $+\infty$, and are not so evaluated by $C_{r}$.

Silverman showed that the criterion for total regularity has the following consequences: No definition of type $G^{\prime}$ possessing an inverse can be equivalent to $I$ both as regards finite and definitely infinite limits unless it is of the form $K: y_{n}=c_{n} x_{n}$, where $\lim _{n \rightarrow \infty} c_{n}=1$. No two definitions $A, B$ of type $G^{\prime}$ possessing inverses can be equivalent both as regards finite and definitely infinite limits unless $A=K B$.

These statements would not hold if the restriction as to the possession of inverses were removed, as may be seen from the trivial example $y_{1}=0 ; y_{n}=x_{n-1}, n>1$; which is equivalent to $I$, even in the present extended sense.

7. Adjunction or Omission of Elements. If a sequence converges, then the new sequence obtained by prefixing or omitting an element at the beginning will converge to the same value. We may inquire whether a similar property holds for definitions of summability; if a sequence is summable $A$ to a value $l$, will the sequence obtained by prefixing or omitting an element be summable $A$ (or summable $B$, where $B$ is expressible by means of $A$ ) to the value $l$ ?

This question has been answered for a number of definitions. In so far as it relates to prefixing an element, the answer must be independent of the value of the element prefixed; only the alteration in rank is significant. The question is equivalent to that of prefixing or omitting a term at the beginning of a series, with appropriate alteration in value of the series; it is in this form that it has usually been studied. 
I shall summarize the most important results. $M^{r}, C_{r}, E_{r}$, whenever they are regular, and $P$, permit adjunction or omission of an element. Borel's "absolute summability," which is not regular, has the same property; a more satisfactory settlement of the question for the Borel means is given by the theorem of Sannia: If a sequence is summable $B_{r}$, then the sequence obtained by omitting an element is summable to the same value at any rate by the stronger definition $B_{r-1}$, and the sequence obtained by omitting an element is summable to the same value even by the weaker definition $B_{r+1}$.

Hardy and Riesz state that it is possible to have a series summable $R_{\lambda, r}$ and remain so summable when a term is omitted, the two values not differing by an amount equal to the omitted term.

8. Necessary Conditions for Summability. If a series $\Sigma u_{n}$ converges, then $u_{n}$ must approach zero; this is of course not sufficient for convergence, but it is a very useful property of convergent series. Somewhat similar necessary conditions exist for summability with respect to the commoner definitions; these conditions are sometimes expressed so as to involve terms of the series, sometimes elements of the sequence. For summability $M^{r}$ or $C_{r}$, a necessary condition is that $\lim u_{n} / n^{r}=0$, and in fact even that $\lim x_{n} / n^{r}=0$; for summability $E_{r}$ it is necessary that $\lim u_{n} /(2 r-1)^{n}=0$ and $\lim x_{n} /(2 r-1)^{n}=0$.

For summability $P$ it is necessary that the series $\Sigma x_{n} t^{n-1}$ and $\Sigma u_{n} t^{n-1}$ have radii of convergence $\geqq 1$, therefore that $\lim \sup \left|x_{n}\right|^{1 / n} \leqq 1$ and $\lim \sup \left|u_{n}\right|^{1 / n} \leqq 1$. For summability $B_{r}$ it is necessary that the series $\Sigma x_{n} t^{n-1} /(n-1) !$ have infinite radius of convergence, hence that $\lim \left|x_{n}\right|^{1 / n} / n=0$ and $\lim \left|u_{n}\right|^{1 / n} / n=0$. A necessary condition that a sequence $\left(x_{n}\right)$ be summable $R_{\lambda, r}$ to $l$ is that

$$
\lim _{n \rightarrow \infty}\left(\frac{\lambda_{n+1}-\lambda_{n}}{\lambda_{n+1}}\right)^{r}\left(x_{n}-l\right)=0 .
$$

9. Multiplication of Series. The totality of expressions obtained by multiplying terms of one series $\Sigma u_{n}$ by terms of another series $\Sigma v_{n}$ may be represented formally by a double series $\Sigma u_{m} v_{n}$. If we collect the terms of this double 
series in any way into a simple series $\Sigma w_{n}$, the latter may be called a product series of the two given series. The behavior of the new series if the original series converges will differ according to the way in which the terms are grouped. Thus, if

$$
\begin{aligned}
& w_{1}=u_{1} v_{1}, \\
& w_{2}=u_{1} v_{2}+u_{2} v_{2}+u_{2} v_{1}, \\
& w_{3}=u_{1} v_{3}+u_{2} v_{3}+u_{3} v_{3}+u_{3} v_{2}+u_{3} v_{1},
\end{aligned}
$$

$\Sigma w_{n}$ will converge whenever $\Sigma u_{n}, \Sigma v_{n}$ converge.

Other methods of grouping, however, are generally more useful. The so-called Cauchy product, suggested by the grouping most natural for power series, takes

$$
\begin{aligned}
& w_{1}=u_{1} v_{1}, \\
& w_{2}=u_{1} v_{2}+u_{2} v_{1}, \\
& w_{3}=u_{1} v_{3}+u_{2} v_{2}+u_{3} v_{1},
\end{aligned}
$$

In this case convergence of $\Sigma u_{n}, \Sigma v_{n}$ does not insure convergence of $\Sigma w_{n}$; it is, however, true that if $\Sigma u_{n}, \Sigma v_{n}$ are convergent, then $\Sigma w_{n}$ will be summable $M$ to the product of the values of $\Sigma u_{n}, \Sigma v_{n}$. More generally, the Cauchy product of two series summable respectively $C_{r}$ and $C_{s}(\mathbf{R}(r)>-1$, $\mathbf{R}(s)>-1)$ is summable $C_{r+s+1}$, and the value of the product series is the product of the values of the given series.

This gives an interesting instance of the use of non-regular definitions. If two series are not merely convergent, but summable $C_{-\frac{1}{2}}$, then their Cauchy product will be convergent.

There is a similar theorem for the Borel-Sannia definition. If two series are summable $B_{r}$ and $B_{s}$, their Cauchy product is summable $B_{t}$ to the correct value, where

$$
t=\left\{\begin{array}{l}
r+s-1 \text { unless } r>0, \text { or } s>0 ; \\
\text { the lesser of } r, s \text { if } r>0, \text { or } s>0 .
\end{array}\right.
$$

The multiplication of Dirichlet's series suggests a different grouping of terms:

$$
w_{n}=\sum_{d} u_{d} v_{n / d},
$$

where $d$ takes as its values the divisors of $n$. For this group- 
ing, which we may call the Riesz product, we have the result: The Riesz product of two series summable $R_{\lambda, r}$ and $R_{\lambda, s}$, where $\lambda_{n}=\log n, r>0, s>0$, is summable $R_{\lambda, r+s+1}$.

10. Extensions to Other Types of Limit. The endeavor to assign a meaning to $\lim _{n \rightarrow \infty} x_{n}$ when it does not exist in the ordinary sense leads naturally to the same attempt for $\lim _{t \rightarrow \infty} x(t)$, where $x(t)$ is a function of the continuous real variable $t$. The analogues of the Cesàro and Hölder means were studied by Landau.* Investigations of the general type corresponding to $G^{\prime}$ were made by Silverman $\dagger$ and Kojima. $\ddagger$ I shall not repeat the most general results of these authors, but shall quote only the following special case:§

If $K(x, y)$ is integrable in $y$ for each value of $x, 0<y \leqq x$, and if for any function $u(x)$ which is bounded and integrable in any finite interval, $x \leqq 0$, we define

$$
v(x)=\alpha u(x)+\int_{0}^{x} K(x, s) u(s) d s,
$$

then a sufficient condition that $\lim _{x \rightarrow \infty} v(x)=l$ whenever $\lim _{x \rightarrow \infty} u(x)=l$ is that for constant $a$,

$$
\int_{0}^{a}|K(x, y)| d y \text { converges, } \quad \lim _{x \rightarrow \infty} \int_{0}^{a}|K(x, y)| d y=0,
$$

that for $x>0$,

$$
\int_{0}^{x}|K(x, y)| d y
$$

is bounded, and that

$$
\lim _{\dot{x} \rightarrow \infty} \int_{0}^{x} K(x, y) d y=1-\alpha .
$$

Extensions to double series have also been made. The analogues of the Cesàro and Hölder means were given by C. N. Moore.\| Robison $\uparrow$ has made a general study of regularity. An element of novelty in comparison to the case of

* SÄChSISCHe Berichte, vol. 65 (1913), p. 131.

$\dagger$ Transactions of this Society, vol. 17 (1916), p. 284; this BuLLETIN, vol. 22 (1915-16), p. 459.

‡ TôHoKu JouRnaL, vol. 14 (1918), p. 64; vol. 18 (1920), p. 37.

$\S$ Silverman, this BuLletin, loc. cit.

|| Transactions of this Society, vol. 14 (1913), p. 73.

If Ph.D. thesis, Cornell, 1919. 
simple series is that even a convergent double series need not have its terms bounded. Robison's result corresponding to the theorem of Silverman and Toeplitz for simple series follows.

$A$ necessary and sufficient condition that the transformation

$$
y_{m n}=\sum_{k=1, l=1}^{m, n} a_{m n k l} x_{k l}
$$

carry every bounded convergent double sequence $\left(x_{m n}\right)$ into a bounded double sequence $\left(y_{m n}\right)$ convergent to the same value is that

$$
\begin{gathered}
\text { for each } k, l, \lim _{m \rightarrow \infty, n \rightarrow \infty} a_{m n k l}=0 ; \\
\text { for all } m, n, \sum_{k=1, l=1}^{m, n}\left|a_{m n k l}\right| \text { is bounded; } \\
\text { for each } l, \lim _{m \rightarrow \infty, n \rightarrow \infty} \sum_{k=1}^{m}\left|a_{m n k l}\right|=0, \\
\text { and for each } k, \lim _{\substack{m \rightarrow \infty, n \rightarrow \infty \\
\lim _{m \rightarrow \infty}}} \sum_{l=1}^{n}\left|a_{m n k l}\right|=0 ; \\
\sum_{k=1, l=1} a_{m n k l}=1 .
\end{gathered}
$$

Robison has given also the theorem for double series corresponding to the result of Carmichael and Hildebrandt, and the condition for total regularity.

11. Other Questions. Time does not permit a detailed account of other interesting lines of study in connection with divergent series; a brief mention of a few results must suffice.

Closely related to the regularity of a transformation of type $G$ is the requirement that it carry every convergent sequence into a convergent sequence (irrespective of any relationship between the two limits); or that it carry a bounded sequence into a convergent sequence, or a bounded sequence into a bounded sequence These conditions have been studied by Kojima, ${ }^{*}$ Fraleigh, $\dagger$ and J. Schur, $\ddagger$ and for double sequences by Robison.|| Interesting investigations have been made of properties possessed not by all series summable according to a certain definition, but only by such of them as satisfy further

* Tônoku Journal, vol. 12 (1917), p. 291.

$\dagger$ A.M. thesis, Cornell, 1918.

‡ Journal FƯr Mathematik, vol. 151 (1920), p. 79.

II Loc. cit. 
conditions. Typical theorems of this kind are the following: If $\Sigma u_{n}$ is summable $M$ and $n u_{n}<K$, then $\Sigma u_{n}$ is convergent. ${ }^{*}$ If $\Sigma u_{n}$ is summable $B_{1}$ and $\sqrt{n}\left|u_{n}\right|<K$, then $\Sigma u_{n}$ is convergent. $\dagger$

For applications of the theory of divergent series to important special types of series, to differential equations, and to mathematical physics, reference may be made to the three expository papers mentioned in $\S 1$.

12. Conclusion. I shall permit myself, in closing, to make two observations which represent only personal opinion.

Any definition of the generalized limit of a sequence is ultimately only an actual limit of something else; it seems to me worth while to recall frequently, in dealing with divergent series, that we are in fact studying only ordinary processes of convergence. It is at times more illuminating for the comprehension of a theorem on summable series to supply all the transformations implied in the definition of summability and state the result entirely in terms of ordinary limits than to use the more concise form which is in essence symbolic. Indeed, important applications of the conditions for regularity are proofs of theorems on limits, in which divergent sequences present themselves, if at all, only as an afterthought. Such applications have been given by Silverman and by Schur.

As regards the various current problems in connection with divergent series, the most important seems to me personally to be that of mutual consistency. It would be desirable to be able to assert of any two definitions which have been used practically that they are or are not mutually consistent, and to have such workable criteria as would make it possible to test new definitions which may be proposed. Without such information, the use of two different methods of summability in a single investigation, unless one is merely included in the other, would seem to produce at least grave inconvenience.

CorNeLl University.

* Hardy, Proceedings of the London Society (2), vol. 8 (1910), p. 302; Landau, PraCe Matematyczno-FizyczNe, vol. 21 (1910), p. 97; Fujiwara, TôнокU JouRNAL, vol. 15 (1919), p. 323.

$\dagger$ Hardy and Littlewood, Rendiconti di Palermo, vol. 41 (1916), p. 36. 